\title{
NEOPLASIAS MALIGNAS PRIMÁRIAS DE APÊNDICE CECAL
}

\author{
PRIMARY MALIGNANT TUMOURS OF THE APPENDIX
}

Affonso A.P. Mércio'; Marcos Weindorfer²; André L. Weber ${ }^{3}$ \& Ana C. Mano ${ }^{4}$

\begin{abstract}
${ }^{1}$ Médico cirurgião; ${ }^{2}$ Médico cirurgião. Ex-residente; ${ }^{3}$ Médico residente - Serviço de Cirurgia Geral; ${ }^{4}$ Acadêmica de Medicina da Universidade Federal de Pelotas, doutoranda - Hospital Nossa Senhora da Conceição - HNSC

CorrespondênciA: Dr. Marcos Weindorfer. Rua Francisco Rodolfo Simch, 680; CEP: 91130-210 - Porto Alegre - RS; Fone (051) $340-0549$
\end{abstract}

MÉRCIO AAP; WEINDORFER M; WEBER AL \& MANO AC. Neoplasias malignas primárias de apêndice cecal. Medicina, Ribeirão Preto, 32: 193-198, abr./jun. 1999.

RESUMO: As neoplasias malignas de apêndice cecal são doenças raras, cujo diagnóstico de certeza é determinado apenas pelo exame histológico da peça. São neoplasias de difícil diagnóstico clínico, não apenas por sua baixa incidência, mas, também, devido a sua forma de apresentação inespecífica, na maioria das vezes, manifestada como apendicite aguda. Os autores revisam a literatura e relatam os resultados obtidos da análise retrospectiva de sete casos de neoplasia maligna de apêndice cecal: cinco casos de tumor carcinóide, um caso de adenocarcinóide e outro de adenocarcinoma. Os casos são a experiência do serviço num período de quatro anos (1994-1997), correspondente a uma incidência de 0,8\% sobre o total de apendicectomias realizadas. São abordados dados referentes a idade, sexo, hipótese diagnóstica pré-operatória, cirurgia realizada e exame anatomopatológico da peça cirúrgica.

UNITERMOS: Adenocarcinoma. Tumor Carcinóide. Neoplasias do Apêndice Cecal.

\section{INTRODUÇÃO}

As neoplasias malignas primárias de apêndice cecal são doenças raras que, quanto à histologia, podem se apresentar como tumores carcinóides, adenocarcinomas ou adenocarcinóides, os últimos apresentando características comuns aos dois outros tipos. Existem algumas controvérsias sobre a classificação dos adenocarcinóides entre os adenocarcinomas, como um subtipo de tumor carcinóide ou como uma entidade separada.

Os tumores carcinóides são neoplasias derivadas de células neuroendócrinas e representam cerca de $80-88 \%$ das neoplasias do apêndice cecal ${ }^{(1,2,3)}$. No trato gastrintestinal, o apêndice é o sitio mais comum de carcinóides, representando cerca de $35-44 \%$ do total. Os sintomas de síndrome carcinóide são raros neste sítio (1\%); e, geralmente, indicam metástase para o fígado ${ }^{(4)}$.
Quanto ao tratamento, como o diagnóstico é, geralmente, feito pelo patologista durante o pós-operatório, cabe ao cirurgião a tarefa, por vezes difícil, de determinar através do laudo anatomopatológico (analisando basicamente diâmetro do tumor, localização no apêndice, idade do paciente e presença de metástases), se o paciente será ou não levado a uma segunda intervenção cirúrgica, desta vez mais agressiva, sob a forma de hemicolectomia direita.

Linfonodos comprovadamente acometidos remetem à hemicolectomia, em qualquer idade ${ }^{(5)}$.

Alguns autores orientam conduta mais agressiva caso o paciente seja criança, pois, nestas, a neoplasia tende a ser mais agressiva ${ }^{(6)}$.

Quanto ao prognóstico, como a grande maioria dos tumores carcinóides apresenta menos de $2 \mathrm{~cm}$ de diâmetro e não tem metástases, a sobrevida em (5) cinco anos chega a 90-100\% ${ }^{(7)}$. O diâmetro do tumor primário, como o mais importante fator prognóstico, foi enfatizado por McGillivray et al. ${ }^{(8)}$. 
O adenocarcinoma de apêndice cecal, outra patologia relatada neste trabalho, é encontrado em cerca de $0,1 \%$ das apendicectomias ${ }^{(1,9)}$, representando 4-6\% de todos os tumores primários do apêndice vermiforme $^{(10)}$. É uma variante rara do câncer de cólon com características próprias ao seu local.

Uma variante da doença é o adenocarcinoma mucinoso, o qual pode estar associado a uma condição denominada pseudomixoma peritone $i^{(11)}$.

Embora quase sempre apresentando-se com quadro clínico de apendicite aguda, por ser uma neoplasia mais agressiva e de caráter mais invasivo que o carcinóide, o adenocarcinoma pode apresentar-se de forma incomum: como massa pélvica com sinais urinários, hidronefrose, infiltração de bexiga, sangramento vaginal, melena e intussuscepção ${ }^{(10,12)}$.

Relatamos ainda um caso, também raro, de tumor adenocarcinóide, por alguns também denominado carcinóide mucinoso, por possuir aparência macroscópica e padrão de tumor carcinóide, mas com o comportamento agressivo do adenocarcinoma ${ }^{(12,13)}$. As características clínicas e histológicas são suficientemente distintas para uma classificação como entidade separada de adenocarcinomas e tumores carcinóides ${ }^{(14)}$.

O prognóstico desta doença é intermediário entre o carcinóide clássico e o adenocarcinoma bem diferenciado $^{(15)}$.

O diagnóstico pré-operatório das neoplasias malignas de apêndice cecal é raramente realizado devido ao seu quadro clínico inespecífico, freqüentemente compatível com um quadro de apendicite aguda. Devem-se conhecer as suas características para que a conduta mais aceitável possa ser tomada em cada situação.

\section{RELATO DE CASOS}

Foram analisados, retrospectivamente, sete (7) casos de neoplasia maligna de apêndice cecal, em pacientes operados de janeiro de 1994 a dezembro de 1997, no Hospital Nossa Senhora da Conceição, em Porto Alegre-RS, período no qual foram realizadas oitocentos e setenta e quatro (874) apendicectomias, com incidência de $0,8 \%$, semelhantes a outros relatos ${ }^{(12,16)}$.

Nos quatro anos estudados, foram tratados cinco casos de tumor carcinóide, um caso de adenocarcinoma e outro de adenocarcinóide. Quatro pacientes eram do sexo masculino e três do sexo feminino. A média geral de idade foi de 41,7 anos (onze (11) a oitenta e quatro (84) anos), sendo que a média de idade nos casos de tumor carcinóide foi de 35,2 anos.
Observou-se que cinco (5) pacientes receberam diagnóstico pré-operatório de apendicite aguda, enquanto outros dois (2) pacientes, de oclusão/suboclusão intestinal.

Em apenas um caso, de tumor carcinóide, houve suspeita transoperatória de neoplasia, devido a um tumor evidente $(2,5 \times 2,5 \times 2,0 \mathrm{~cm})$, com invasão de ceco, coloração amarelada e aderências locais, realizando-se hemicolectomia direita no mesmo procedimento (Figura 1).

Dentre os demais seis casos, um foi submetido também a hemicolectomia direita, um mês após apendicectomia, com base no exame histológico do apêndice cecal, compatível com adenocarcinoma moderadamente diferenciado, com invasão de serosa - Dukes B (Figura 2); os outros cinco casos foram submetidos apenas a apendicectomia, sendo quatro (4) tumores carcinóides, variando de 1 a $2 \mathrm{~cm}$ de diâmetro e distantes da margem cirúrgica, e um pequeno foco de neoplasia adenocarcinóide na ponta do apêndice vermiforme (Figura 3).

\section{DISCUSSÃO}

É importante, na avaliação clínica dos pacientes, lembrar que as neoplasias malignas de apêndice cecal, indiferentemente do tipo histológico, apresentam-se, na maioria dos casos, com clínica de apendicite aguda (cerca de 68\%), provavelmente devido à obstrução maligna da luz apendicular, ocasionando infecção sobreposta ${ }^{(6,12,17,18)}$. Em nenhum dos casos, aqui relatados, houve suspeita pré-operatória de neoplasia de apêndice cecal e, na maioria dos casos, o paciente foi levado à cirurgia sob hipótese diagnóstica de apendicite aguda.

O diagnóstico de certeza da neoplasia e seu tipo histológico é conferido apenas pelo exame histológico da peça.

\section{TUMORES CARCINÓIDES}

Os cinco (5) casos relatados de tumor carcinóide representam $0,57 \%$ das apendicectomias realizadas no período, coincidindo com o percentual descrito na literatura ${ }^{(19)}$. Nosso estudo apontou uma média de idade de 35,2 anos (onze (11) a oitenta e quatro (84) anos), enquanto a literatura relata que a neoplasia se manifesta em todas as idades, com idade média de trinta e dois (32) anos ${ }^{(6)}$. 


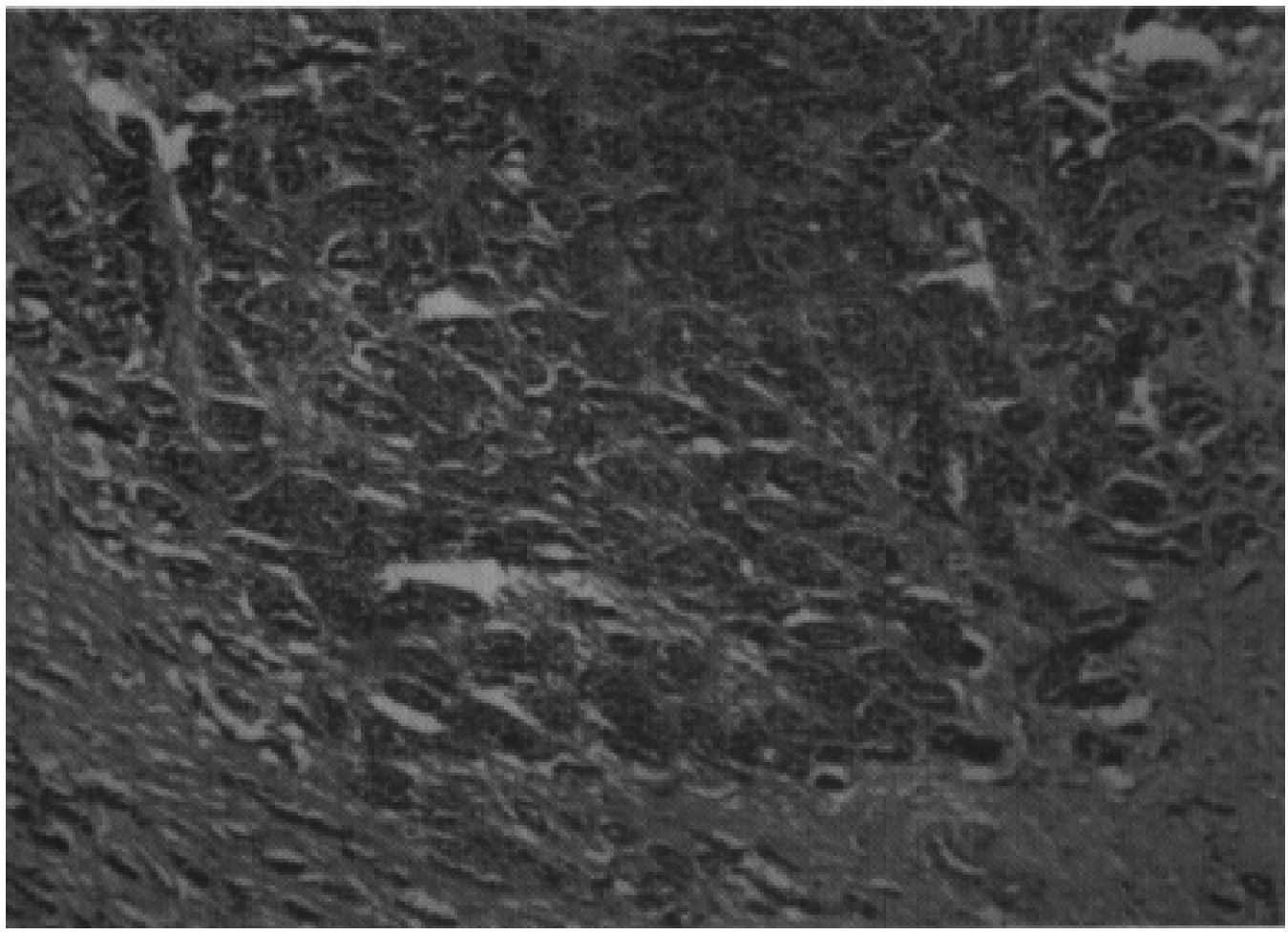

Figura 1 - Tumor carcinóide de apêndice cecal.

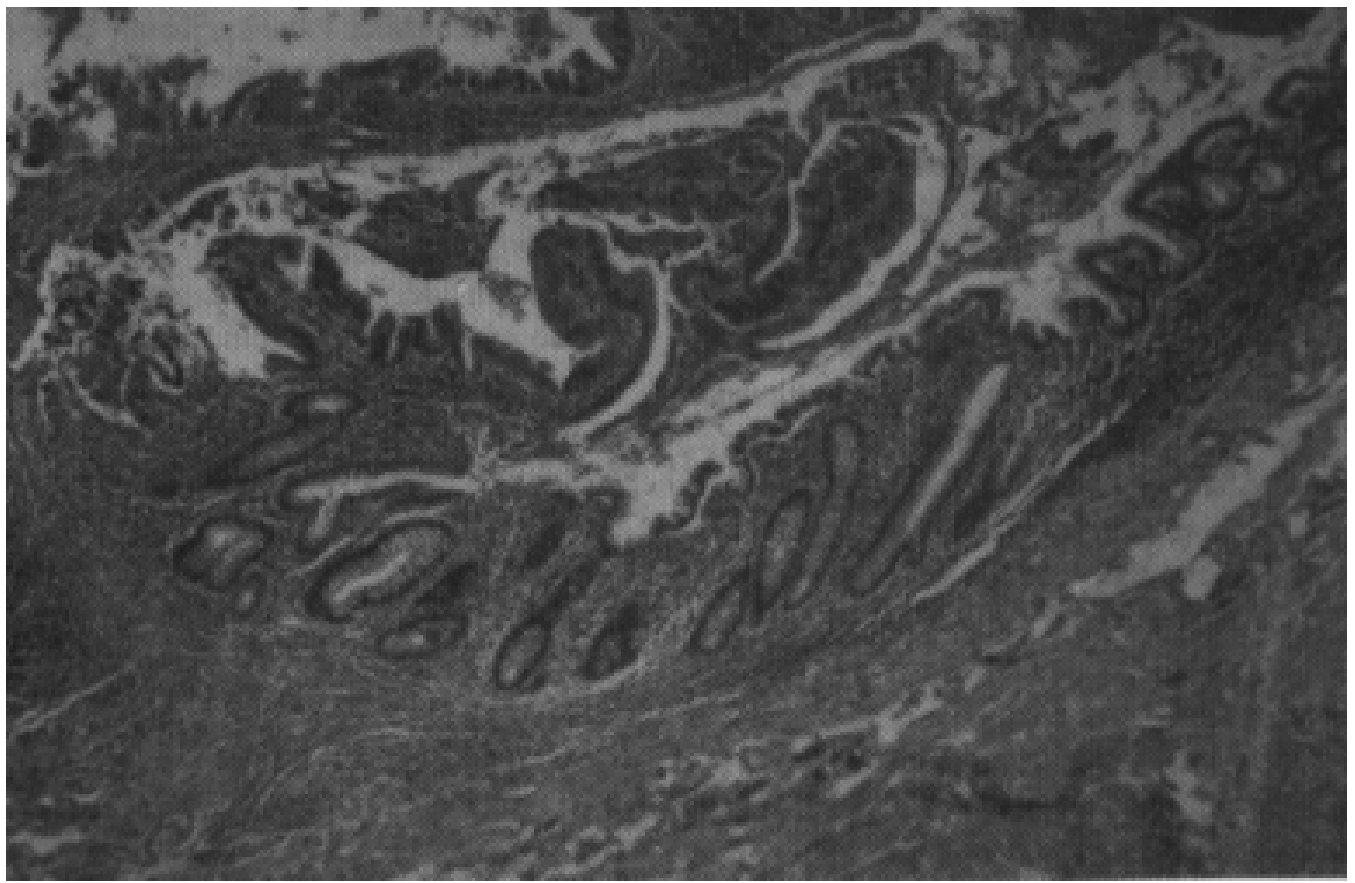

Figura 2 - Transição entre o tecido glandular normal e o adenocarcinoma (acima), o qual se projeta na luz apendicular. 


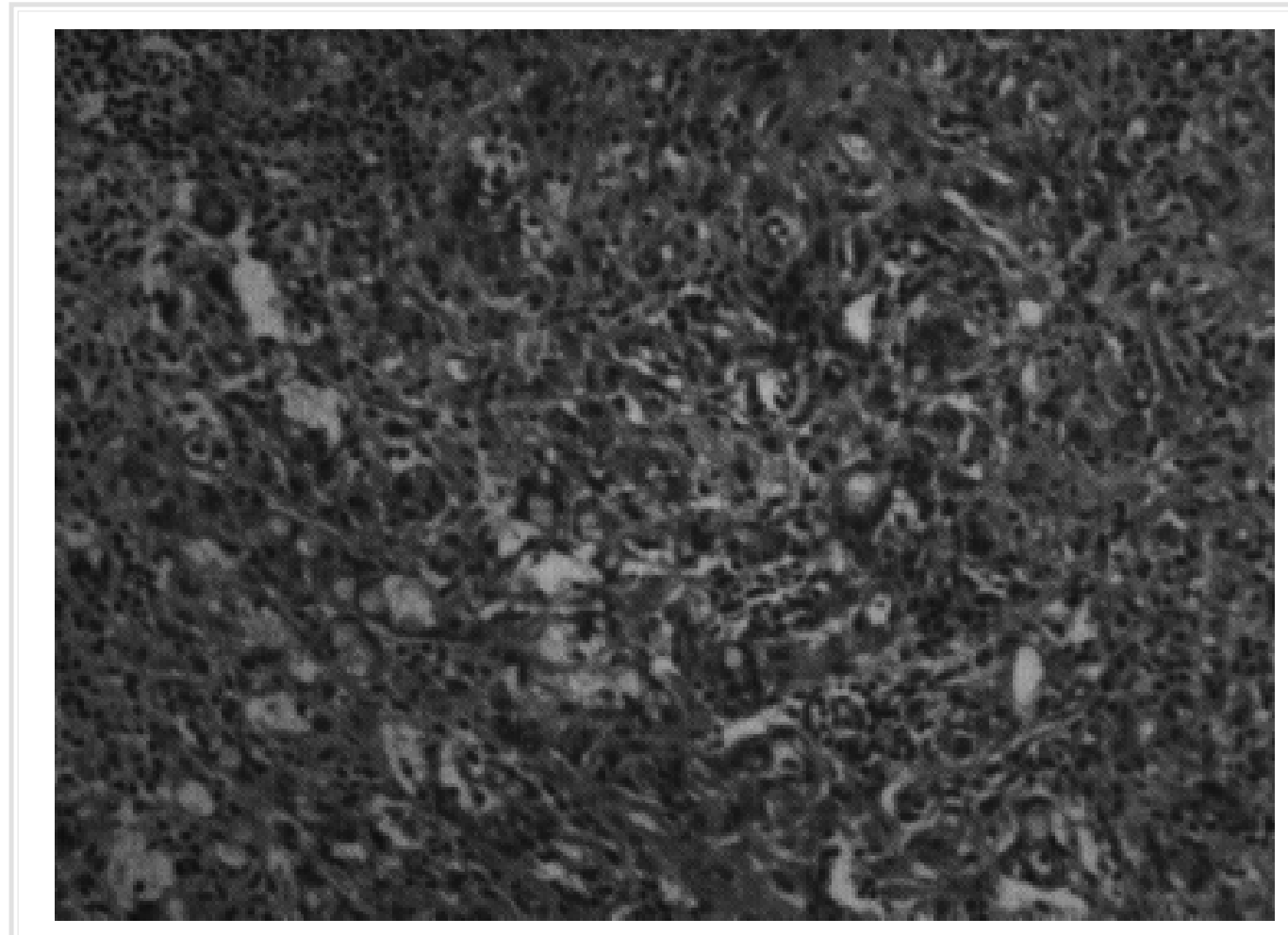

Figura 3 - Adenocarcinóide de apêndice cecal.

Carcinóides são descritos como mais comuns em mulheres que em homens, na proporção de $1: 4^{(2,6,17)}$. Os casos descritos neste trabalho são representados por três (3) mulheres e dois (2) homens. Aproximadamente $80 \%$ dos tumores carcinóides medem menos de um (1) centímetro; $14 \%$ entre 1 e $2 \mathrm{~cm}$ e $6 \%$ maior do que $2 \mathrm{~cm}^{(6,17)}$. Em nosso estudo, quatro tumores mediam de 1 a $2 \mathrm{~cm}$, distantes da margem cirúrgica, e foram submetidos a simples apendicectomia, enquanto apenas um tumor apresentava-se com mais de $2 \mathrm{~cm}$ de diâmetro e invadindo o ceco, sendo realizada hemicolectomia direita.

De acordo com a literatura, a simples apendicectomia está adequada para todos os casos com apêndice com diâmetro inferior a $1 \mathrm{~cm}$ e para a maioria das neoplasias com diâmetro de 1-2 cm.

Moertel et al. ${ }^{(5)}$, que seguiram, por um longo período, um grande número de pacientes após simples apendicectomia por tumores carcinóides, relatam que nenhum paciente que apresentou tumor menor que $2 \mathrm{~cm}$ de diâmetro morreu por recorrência tumoral ou metástase. Indicações de intervenção mais agressiva são, além do diâmetro da massa, a extensão do tumor no mesoapêndice, localização na base do apêndice, invasão linfática subserosa e idade do paciente. Estes critérios para cirurgia mais ampla, aplicam-se bem no momento de decidir sobre realização de apendicectomia ou hemicolectomia direita em pacientes com neoplasias de $1-2 \mathrm{~cm}^{(20)}$.

Considera-se que a extensão da invasão tumoral, ao contrário de carcinóides do íleo, estômago e cólon, é de pouco valor preditivo para o potencial metastático, importando mais o tamanho do tumor. Em nenhum dos pacientes descritos em nosso relato, observou-se presença de metástases.

$\mathrm{O}$ risco de metástases em tumores medindo menos de $1 \mathrm{~cm}$ de diâmetro é nulo; em tumores de $1-2 \mathrm{~cm}$ é de $0-11 \%$ e em tumores com mais de $2 \mathrm{~cm}$, o risco de metástases é consideravelmente maior, entre 30 e $60 \%{ }^{(5,21)}$.

\section{ADENOCARCINOMA}

Esta doença é mais comum entre a quinta $\left(5^{\mathrm{a}}\right)$ e sexta $\left(6^{\mathrm{a}}\right)$ décadas de vida, sem predominância quan- 
to ao $\operatorname{sexo}^{(9,10,12,22)}$. Nosso caso é representado por um homem de cinqüenta e três (53) anos de idade.

Seu tratamento é cirúrgico, sendo tradicionalmente citado que a apendicectomia é suficiente para tratar lesões não invasivas (restritas à mucosa, o que é, por vezes, difícil determinar), e que lesões invasivas deveriam ser tratadas por hemicolectomia direita ${ }^{(3,9)}$, tal como em nosso caso.

Trabalhos recentes sugerem que o tratamento deveria ser sempre hemicolectomia, mesmo que realizado secundariamente ${ }^{(23)}$. Hesketh ${ }^{(24)}$ demonstrou que, na presença de invasão tumoral, a apendicectomia seguida de hemicolectomia direita aumenta a sobrevida em cinco (5) anos para 63\%, sendo que, na apendicectomia apenas, é de $21 \%$. A quimioterapia adjuvante pode ser utilizada de acordo com os critérios estabelecidos para o câncer de cólon ${ }^{(5)}$. Nosso paciente não foi submetido a nenhum tratamento adjuvante.

O prognóstico do adenocarcinoma primário de apêndice cecal depende do grau de diferenciação e do estadiamento de Dukes ${ }^{(22,25,26)}$. No caso apresentado, o exame histológico revelou extensão de neoplasia moderadamente diferenciada ao tecido adiposo pericecal, comprometendo-o, mas sem metástase em linfonodos, caracterizando um estadiamento Dukes B. Pacientes com estágio Dukes B têm sobrevida, em cinco anos, estimada em $67 \%{ }^{(23)}$.

Não houve perfuração do apêndice cecal no caso relatado. Na literatura, alguns autores afirmam que ocorre perfuração em cerca de $55 \%$ dos casos de neoplasia de apêndice cecal, sendo, portanto, o sítio mais freqüente de perfuração do trato gastrintesti- nal $^{(3,23)}$; além de que isto poderia determinar um pior prognóstico pela possibilidade de disseminação de células neoplásicas ${ }^{(27)}$. Outros autores, no entanto, analisando séries mais numerosas, não demonstram diferença estatisticamente significativa quanto à alteração do prognóstico no caso de perfuração (diferente das outras neoplasias do trato gastrintestinal com perfuração), além de que a perfuração possa até favorecer um diagnóstico precoce ${ }^{(3,23)}$.

\section{ADENOCARCINÓIDE}

A média de idade, em uma metanálise de cento e oito (108) casos, é de 51,5 anos, com igual distribuição quanto ao sexo ${ }^{(14)}$. O caso apresentado corresponde a um homem de sessenta e três (63) anos.

Macroscopicamente, os adenocarcinóides apresentam-se tipicamente como um espessamento fibroso do apêndice, tal como nosso caso, e não como nódulo. Estas neoplasias são melhor tratadas por hemicolectomia direita, se preenchem uma ou mais das seguintes características: evidência de infiltração difusa no apêndice; foco microscópico atípico ou presença de duas ou mais mitoses por dez (10) campos de grande aumento (X400, ocasionando um campo de $0,23 \mathrm{~mm}^{2}$ ). Caso tais características não sejam observadas, como em nosso relato, a apendicectomia, apenas, está adequada ${ }^{(13,14)}$.

Warkel et al. ${ }^{(13)}$, em uma série de trinta e nove (39) casos, identificaram metástase em $17,1 \%$ dos pacientes; já Wolff \& Ahmed ${ }^{(26)}$, em uma série de doze (12) pacientes, verificaram metástase em um terço de seus pacientes.

MÉRCIO AAP; WEINDORFER M; WEBER AL \& MANO AC. Primary malignant tumours of the appendix. Medicina, Ribeirão Preto, 32: 193-198, apr./june 1999.

ABSTRACT: The malignant tumours of the appendix are unusual neoplasms, which definitive diagnosis is made only by histological examination of the specimen. Preoperative diagnosis is difficult due to the inespecific presentation, usually presented as acute appendicitis. The authors review the literature and report a retrospective analysis of seven cases of malignant tumour of the appendix: five carcinoid, one adenocarcinoid and one adenocarcinoma. These cases are the Hospital Nossa Senhora da Conceição experience from 1994 to 1997, and denote an incidence of $0,8 \%$, among all appendectomies. The study analyses patients age, sex, preoperative diagnosis, operative procedure and histological exam of the specimen.

UNITERMS: Adenocarcinoma. Appendiceal Neoplasms. Carcinoid Tumor. 


\section{REFERÊNCIAS BIBLIOGRÁFICAS}

1 - GUPTA SC et al. Pathology of tropical appendicitis. J Clin Pathol 42: 1169-1172, 1989.

2 - LAMPERTICO P. Clinicopathologic considerations on 9952 appendices removed at the Busto Arsizio Hospital from 1966 to 1989. Pathologica 82: 719-732, 1990.

3 - CERAME MA. A 25-year review of adenocarcinoma of the appendix. Dis Colon Rectum 31: 145-150, 1988.

4 - CRAWFORD JM. The gastrointestinal tract. In: ROBBINS SL, ed. Pathologic basis of disease, $5^{\text {th }}$ ed, W.B. Saunders, Philadelphia, p. 818-820, 1994.

5 - MOERTEL CG et al. Carcinoid tumor of the appendix: Treatment and prognosis. N Engl J Med 317: 1699-1701, 1987.

6 - ROGGO A; WOOD WC \& OTTINGER LW. Carcinoid tumors of the appendix. Ann Surg 217: 385-390, 1993.

7 - GODWIN JD. Carcinoid tumors. An analysis of 2.837 cases. Cancer 36: 560-569, 1975.

8 - MACGILLLIVRAY DC et al. Distant metastasis from a carcinoid tumor of the appendix less than one centimeter in size. Surgery 111: 466-471, 1992.

9 - BURGESS P \& DONE HJ. Adenocarcinoma of the appendix. J R Soc Med 82: 28-29, 1989.

10 - CORTINA R et al. Management and prognosis of adenocarcinoma of the appendix. Dis Colon Rectum 38: 848-852, 1995.

11 - JASS JR. Tumour of the small and large intestines. In: FLETCHER CC, ed. Diagnostic histopathology of tumors, Churchill Livingstone, New York, v. 1, p. 251-253, 1995.

12 - DEANS GT \& SPENCE RAJ. Neoplastic lesions of the appendix. Brit J Surg 82: 299-306, 1995.

13 - WARKEL RL; COOPER PH \& HELWIG EB. Adenocarcinoid, a mucin-producing carcinoid tumor of the appendix: a study of 39 cases. Cancer 42: 2781-2793, 1978.

14 - BAK M \& ASSCHENFELDT P. Adenocarcinoid of the vermiform appendix: a clinicopathologic study of 20 cases. Dis Colon Rectum 31: 605-612, 1988.
15 - ANDERSON NH et al. Appendiceal goblet cell carcinoids: a clinicopathological and immunohistochemical study. Histopathology 18: 61-65, 1991.

16 - MACEDO AR; ARAÚJO JR \& FREITAS JG. Tumores malígnos primários do apêndice vermiforme: Estudo clínico patológico de 8 casos. Arq Gastroenterol 16: 62-66, 1979.

17 - JONSSON T; JOHANNSSON JH \& HALLGRIMSSON JG. Carcinoid tumors of the appendix in children younger than 16 years. A retrospective clinical and pathologic study. Acta Chir Scand 155: 113-116, 1989.

18 - LESNICK G \& MILLER D. Adenocarcinoma of the appendix. Cancer 2: 18-24, 1949.

19 - STORER EH. Appendix. In: SCHWARTZ SI, ed. Principles of surgery, $2^{\text {nd }}$ ed, McGraw-Hill, New York, p. 1167-1176, 1969.

20 - ROTHMUND M \& KISKER O. Surgical treatment of carcinoid tumors of the appendix, colon and rectum. Digestion 55: 86-91, 1994. Suppl 3.

21 - THIRLBY RC; KASPER CS \& JONES RC. Metastatic carcinoid tumor of the appendix: Report of a case and review of the literature. Dis Colon Rectum 27: 42-46, 1984.

22 - TRIPODI $\mathrm{J}$ et al. Primary adenocarcinoma of the appendix: an unusual presentation. Am J Gastroenterol 90: 661-662, 1995.

23 - NITECKI SS et al. The natural history of surgically treated primary adenocarcinoma of the appendix. Ann Surg 219: 51-57, 1994.

24 - HESKETH KT. The management of primary adenocarcinoma of the veriform appendix. Gut 4: 158-168, 1963.

25 - LISS AP. Appendiceal malignancies. Semin Oncol 15: 129-137, 1988.

26 - WOLFF M \& AHMED N. Epithelial neoplasms of the veriform appendix (exclusive of carcinoid). Cancer 37: 2493-2510, 1976.

27 - BROWN HW \& HUSNI EA. Ruptured adenocarcinoma of the appendix: review of the literature and case presentations. Surgery 42: 953-959, 1957.

Recebido para publicação em 05/10/98

Aprovado para publicação em 01/06/99 